\title{
AN ANALYSIS OF SOLIDIFICATION PATH IN THE NI-BASE SUPERALLOY, CMSX10K
}

\author{
N. D’Souza ${ }^{1}$, H. B. Dong ${ }^{1,2}$ \\ ${ }^{1}$ Rolls-Royce plc, PO. Box 31, Derby DE24 8BJ, UK \\ ${ }^{2}$ Department of Engineering, University of Leicester, University Road, Leicester LE1 7RH, UK
}

Keywords: Solidification, Micro-Segregation, Inter-Dendritic, CMSX10K

\begin{abstract}
A detailed analysis of solidification path has been conducted in a typical $3^{\text {rd }}$ generation Ni-base superalloy, CMSX10K. Using calorimetry and X-ray spectroscopy the solidification sequence, pertinent phase transformation temperatures, evolution of fraction solid with temperature and the accompanying micro-segregation has been determined. Particular emphasis is directed at the latter stages of solidification when non-equilibrium phases form within the inter-dendritic regions. A detailed characterisation of this constituent is carried out to highlight the severity of microsegregation. Relevance and applications of these results in the design of optimum heat treatment cycles to eliminate the as-cast coring and also guidelines from the view of alloy design and its implications to castability are discussed.
\end{abstract}

\section{Introduction}

Ni-base superalloys have found increasing applications in turbine components for aero-engines as well as land-based power generation. The high temperature strength is derived from the $\gamma$ precipitates in addition to solid solution strengthening within the $\gamma$ matrix. The increasing requirements for sustained high temperature strength has led to a continued focus on developing newer generation superalloys, where micro-structural stability at high temperature has conferred enhanced creep and fatigue strength to the turbine components [1].

The modern generation $\left(2^{\text {nd }}, 3^{\text {rd }}\right.$ and $\left.4^{\text {th }}\right)$ Ni-base superalloys have been characterized by increased refractory additions, such as Ta, $\mathrm{W}$ and $\mathrm{Re}[2]$. While these elements improve high temperature strength, their sluggish diffusivity in the solid-state leads to significant segregation and the formation of the highly cored eutectic microstructure during solidification. Consequently, a heat treatment process is required, which is aimed at: (a) Dissolution of the inter-dendritic constituent and attainment of compositional homogeneity across the dendrite cross-section and (b) Following step (a), the component is heated to above the $\gamma^{\prime}$ solvus temperature and then rapidly quenched and subsequently aged at an intermediate temperature such that the desired cubic morphology of the $\gamma^{\prime}$ precipitates is produced. It is this microstructure that imparts the elevated temperature strength. There are specific features of interest that arise. Specifically, to minimize the cost of heat treatment, it is important to deduce the optimum time / temperature sequence, especially with regards to step (a). This requires a detailed characterisation of the interdendritic constituent. While dissolution of the non-equilibrium phases and subsequent homogenization can be achieved by heating to below the incipient melting point and purely relying on solid-state diffusion, a more innovative approach that can appreciably decrease the solutioning time is by heating above the incipient melting point to produce localized melting within the inter-dendritic region [3, 4]. By subsequent holding at this temperature, the resultant melted region is "healed" and liquid disappears with growth of the primary solid and is similar in principle to the phenomenon of constitutional liquation [5]. The principal advantage is aimed at employing liquid-state diffusion to aid in the dissolution of the non-equilibrium phases, given that liquid-state diffusion is several orders of magnitude greater than diffusion in the solid-state.

Therefore, it is imperative to determine: (1) the solidification sequence and the appropriate temperatures that correspond to the freezing of phases within the inter-dendritic constituent and (2) the extent of occurrence of the inter-dendritic constituent within the microstructure before an appropriate time / temperature cycle can be designed. This forms the emphasis of this article. The alloy studied was CMSX10K (nominal composition, Table I).

Table I. Nominal Composition in Weight Percent (wt \%) of the Ni - Base Superalloy CMSX 10K

\begin{tabular}{rrrrrrrrrrc}
\hline Chemistry & Al & Ti & Cr & Co & Mo & Nb & Ta & W & Re & Ni \\
\hline CMSX 10K & 5.8 & 0.2 & 2.3 & 3.3 & 0.4 & 0.1 & 8.3 & 5.5 & 6.3 & Bal \\
\hline
\end{tabular}

\section{Experiments}

\section{Differential Scanning Calorimetry (DSC)}

A Netzsch 404 Differential Scanning Calorimeter (DSC) was used to determine the enthalpy and heat capacity from the measured raw DSC data. Consequently, the phase transition temperatures and the evolution of fraction solid with temperature were calculated. Cylindrical samples (diameter $4 \mathrm{~mm}$, height $3 \mathrm{~mm}$ ) machined from the CMSX10K bar stock were used. The DSC unit was calibrated using pure $\mathrm{Au}$ and $\mathrm{Ni}$ and all experiments were conducted using a dynamic high purity Ar atmosphere to minimise potential oxidising effects. The samples were subjected to heating / cooling cycles as follows:

Room temperature $\rightarrow$ heating $\left(25 \mathrm{~K} \mathrm{~min}^{-1}\right) 1000^{\circ} \mathrm{C} \rightarrow$ isothermal holding at $1000^{\circ} \mathrm{C}$ for 10 minutes $\rightarrow$ heating $\left(5 \mathrm{~K} \mathrm{~min}^{-1}\right) 1400^{\circ} \mathrm{C}$ $\rightarrow$ isothermal holding at $1400^{\circ} \mathrm{C}$ for 10 minutes $\rightarrow$ cooling $\left(5 \mathrm{~K} \mathrm{~min}^{-1}\right) 1000^{\circ} \mathrm{C} \rightarrow$ isothermal holding at $1000^{\circ} \mathrm{C}$ for 10 minutes $\rightarrow$ cooling $\left(25 \mathrm{~K} \mathrm{~min}^{-1}\right)$ room temperature.

The cooling rate chosen $\left(5 \mathrm{Kmin}^{-1}=0.08 \mathrm{~K} \mathrm{~s}^{-1}\right)$ was typical of conditions encountered during industrial directional solidification. The raw DSC data (heating / cooling) was subsequently manipulated using a numerical programme $\boldsymbol{C A L C O R}$ to calculate the enthalpy change from the DSC measurements by treating the complete process of heat flow within the DTA apparatus, details of which have been reported elsewhere [6]. 


\section{$\underline{\text { Microscopy - Optical and Scanning Electron (SEM) }}$}

Transverse sections, which were cut orthogonal to the casting axis from single crystal test bars directionally solidified, were examined to study the as-cast microstructure. The test bars were directionally solidified in an industrial furnace and details of the casting furnace are available elsewhere [7].

Samples for micro-structural and compositional characterisation were performed using scanning electron microscopy. A Gemini LEO 1525 FEG-SEM was used. An operating voltage of $20 \mathrm{kV}$ and a working distance of $15 \mathrm{~mm}$ were the principal operating parameters. For routine imaging, the secondary electron and backscattered imaging modes were used. To reveal the inter-dendritic morphology in greater depth, a deep etch method was adopted. The etchant used had a composition by volume percentage as follows: Nitric acid - $33 \%$, Acetic acid - $33 \%$, Hydrofluoric acid - $1 \%$, Distilled water - $33 \%$. The etch time was 30 seconds. Imaging in the latter case was performed using the InLens detector. This is a type of secondary electron detector that is mounted in the pole piece (much closer to the sample surface than a normal secondary electron detector) and has a higher efficiency at lower accelerating voltages. In this instance, the accelerating voltage used was $5 \mathrm{kV}$ and the working distance was $5 \mathrm{~mm}$.

Additionally, compositional analyses were performed on flat aspolished surfaces using a Cameca SX100 Electron Probe MicroAnalyser (EPMA). A beam accelerating voltage of $20 \mathrm{kV}$ was used, with a beam current of $10 \mathrm{nA}$ for $\mathrm{Ni}$ analysis and $100 \mathrm{nA}$ for analysis of all other elements. The beam was focused to a diameter of $1 \mu \mathrm{m}$. Calibration standards were all pure metals (99.999\% or better), except for aluminium for which $\mathrm{Al}_{2} \mathrm{O}_{3}$ was used. Counting times on the background were half that at the peak. Background positions and slope factors to avoid background overlaps affecting Mo, W, Re and Ta were calculated using Virtual WDS. X-ray data processing used Cameca's PeakSight software, applying the XPhi data reduction method.

\section{Details for Compositional Analysis}

For compositional analysis, the as-cast sample coupons were heated in an industrial heat treatment furnace (BodyCote Ltd., Derby, UK) at $10 \mathrm{~K} \mathrm{~min}^{-1}$ from ambient to the incipient melting point $\left(1358^{\circ} \mathrm{C}\right.$ and determined using calorimetry) and then quenched. The resulting microstructure is comprised of a very fine $\gamma^{\prime}$ precipitate distribution across the entire dendrite cross-section. Compositional analyses were performed on these transverse sections. The rationale for this approach is reported in the results section. The composition measurements were comprised of two types:

1. At dendrite cores, spot measurements were conducted over a series of dendrite cores that were easily identified on transverse sections, since the axial orientation of the test bars were within $8^{\circ}$ from $<001>$.

2. At periphery of the secondary dendrites, spot measurements were also conducted along the periphery of the secondary lobes. The spatial location of the measurement points on the dendrite cross-section was in close proximity to the lobe perimeter. The shortest distance of any measurement point was $5 \mu \mathrm{m}$ from the lobe periphery; this will be discussed in a latter section.

\section{Results and Discussions}

\section{Phase Transformations and as-cast Microstructure}

The measured DSC cooling curves have been manipulated using CALCOR as mentioned in section 2.1 to calculate the variation of enthalpy $(H)$ and effective heat capacity $\left(C_{E P}=\frac{d H}{d T}\right)$ with sample temperature, $T$; these are shown in Figs. 1a, b respectively. Phase transition temperatures were obtained from the $C_{E P}$ curve; the start of transformation corresponds to the onset of the exotherm for cooling curves. In this study we use the cooling curve to determine the liquidus following the enthalpy method proposed by Dong and Brooks [8]. Accordingly:

i. $\quad$ Solidification commences at $\mathrm{T}=1406^{\circ} \mathrm{C}$. This is defined as the onset temperature. A nucleation undercooling of $5 \mathrm{~K}$ was measured from the observed recalescence. This therefore gives a liquidus temperature of $\mathrm{T}_{\mathrm{Liq}}=1411^{\circ} \mathrm{C}$.
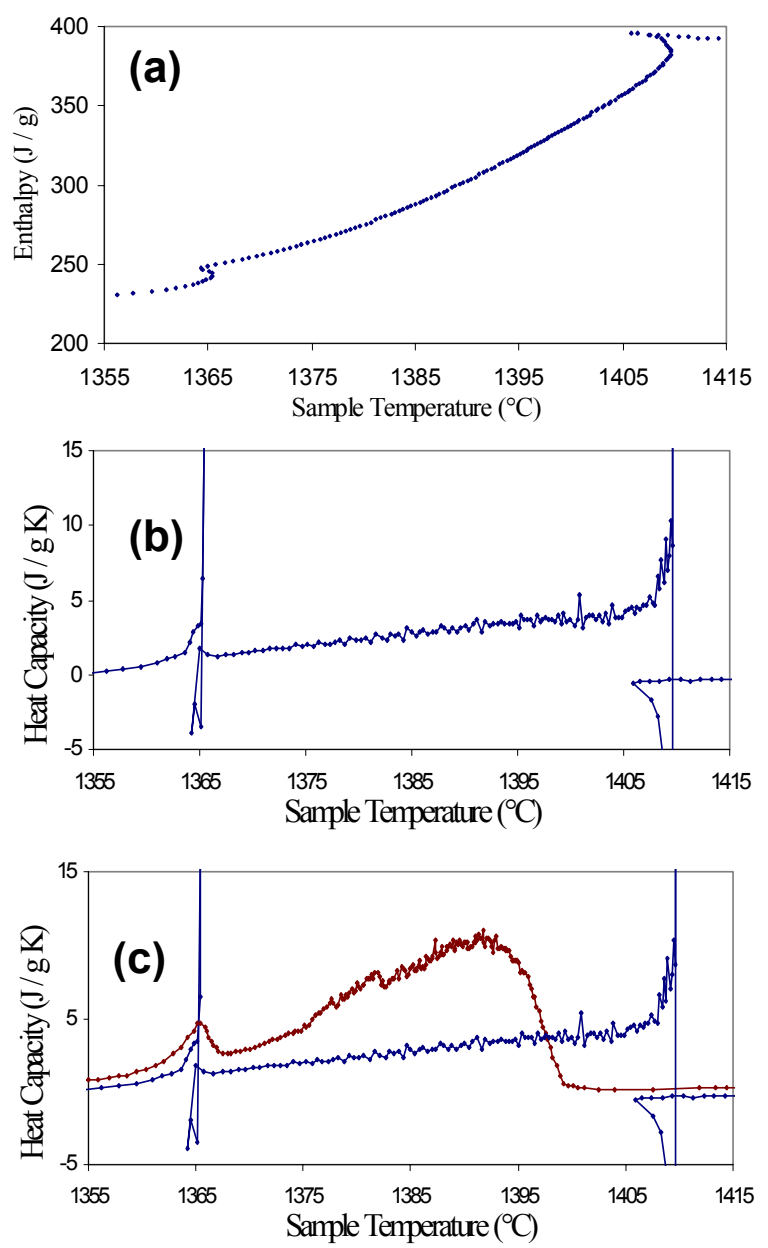

Fig. 1 Differential Scanning Calorimeter (DSC) results: (a) Enthalpy versus temperature (Cooling), (b) Effective heat capacity versus temperature (Cooling), (c) Effective heat capacities for heating and cooling cycles superimposed. 
ii. Solidification is then accompanied by decreasing enthalpy.

iii. A second sharp transition was observed at $\mathrm{T}=1365^{\circ} \mathrm{C}$. We associate this with the onset of freezing of the nonequilibrium phases.

In Fig. 1c, the corresponding effective heat capacity curves for the heating and cooling cycles are superimposed. The two curves show similarity at lower temperatures, but at increasing fraction liquid during melting appreciable deviation occurs and this has also been observed in other studies in Ni-base superalloys and has been attributed to diffusion $[9,10]$. Solidification terminates at the solidus temperature and a very good correlation was observed between the heating and cooling curves; $T_{\text {Solidus }}=1358^{\circ} \mathrm{C}$ and this was determined by inspecting the deviation of the enthalpy curve from the base line in an enlarged view in both cases.

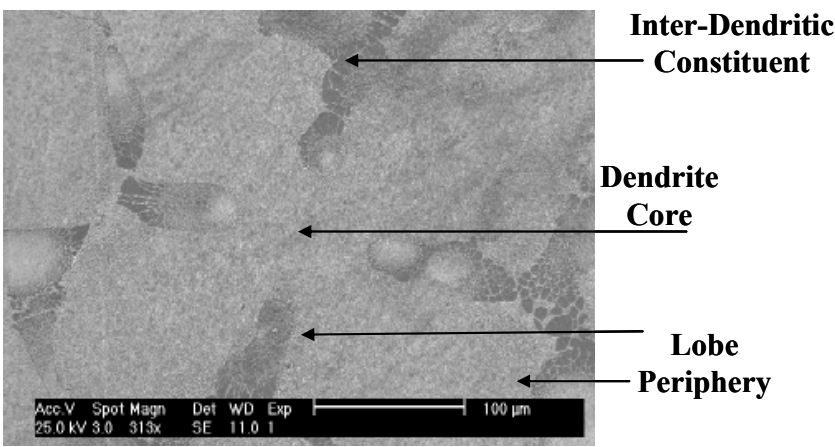

Fig. 2 Representative back-scattered electron image (BEI) showing dendrite cross-section with core and periphery of secondary lobes and inter-dendritic region

Fig. 2 is a typical back-scattered electron image (BEI) that shows the dendrite microstructure in detail. The contrast arises from preferential solute partitioning. The cruciform dendrite lobes are seen and also visible is the inter-dendritic constituent that is clearly distinguished from the dendrites. Thermodynamic calculations using the EQLB (equilibrium - complete mixing in solid and liquid) solidification models in a commercial software $\mathrm{JMatPro}^{\mathrm{TM}}$ have been carried out. JMatPro ${ }^{\mathrm{TM}}$ uses a multicomponent thermodynamic database, Ni-Data developed by ThermoTech Ltd (UK) [11]. In the EQLB model, the solidification product is only the $\gamma$ phase and the transformation can be represented as: $\mathrm{L} \rightarrow \gamma$, i.e. only primary solidification occurs. However, inspection of Fig. 2 clearly shows that this is not the case and solidification progresses beyond the primary stage and terminates with the formation of the inter-dendritic constituent, which constitutes the non-equilibrium phase mixture.

\section{Implications of Micro-segregation}

The restricted diffusion in the solid is the cause of microsegregation, leading to composition gradients within the solid. A schematic binary phase diagram is shown in Fig. 3, where the liquidus and solidus are indicated and presented as linear for simplicity. However, lack of mixing in the solid leads to a depressed solidus, because of which solidification proceeds below the equilibrium solidus temperature and depending on the extent of segregation, the local liquidus temperature can eventually reach the invariant (eutectic) reaction point and leading to the formation of non-equilibrium eutectic. It should be noted that equilibrium is always maintained at the S / L interface and at any temperature the liquid and solid compositions move along the liquidus and solidus lines respectively; however the average composition moves along the depressed solidus.

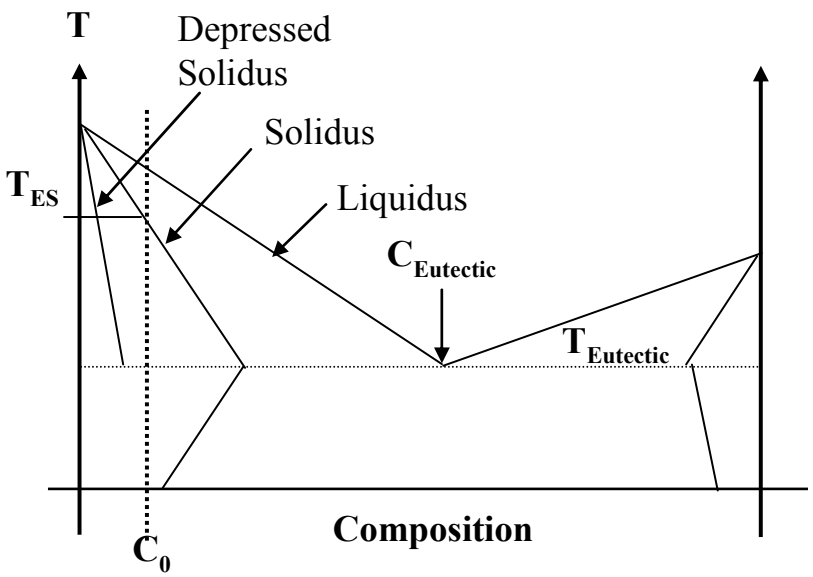

Fig 3 A schematic binary phase diagram showing the liquidus and solidus, equilibrium solidus temperature $\left(T_{E S}\right)$ and depressed solidus

\section{Segregation across Dendrite Cross-section}

The BEI in Fig. 2 shows the dendrite cross-section. In earlier studies involving detailed compositional analysis using X-ray spectroscopy to characterize the as-cast micro-segregation, it was shown that the composition measurements were distinctly biased by the $\gamma / \gamma^{\prime}$ distribution within the X-ray interaction volume [12]. The $\gamma^{\prime}$ precipitates within the dendrites nucleate in the $\gamma$ matrix below the $\gamma^{\prime}$ solvus temperature in the solid-state. Significant scatter in the measured composition was observed at regions having solidified at the same temperature, such as the periphery of the dendrite lobes, i.e. bordering the inter-dendritic region. The extent of scatter across such regions is therefore an artefact and must be identified and should be delineated from the experimentally measured as-cast segregation. The extent of scatter was governed by the size and distribution of the $\gamma / \gamma^{\prime}$ phases in relation to the interaction volume. The important operational parameters therefore were the probe size, the accelerating voltage and the X-ray line used, which have a direct bearing on the dimensions of the interaction volume from where the X-rays are generated.

One of the ways in overcoming the $\gamma / \gamma^{\prime}$ volume averaging effects in compositional measurements, is to produce a uniform $\gamma / \gamma$ distribution in relation to the interaction volume and it has been shown previously that this can be achieved by producing a fine $\gamma / \gamma /$ distribution across the dendrite cross-section. This can be achieved as follows:

- Heating to above the $\gamma^{\prime}$ solvus temperature - this dissolves the $\gamma$ precipitates in the $\gamma$ matrix.

- $\quad$ Rapid quench - $\gamma^{\prime}$ precipitates and the quench inhibits growth of $\gamma^{\prime}$ resulting in a fine $\gamma / \gamma^{\prime}$ distribution. 
In these experiments, we heat the specimen to the incipient melting point and quench (section 2.3). Any considerations of solid-state diffusion during the heating cycle can be ignored, given the sluggish diffusivity of the refractory elements (Ta, W, $\mathrm{Re})$ and the rapid heating rates involved. This is borne out in Fig. 1c, where very little difference is observed between the heating and cooling effective heat capacity curves at temperatures in the vicinity of the incipient melting point.

Keeping in mind the role of volume averaging and implications of micro-segregation as highlighted in Fig. 3, we have therefore chosen: (i) dendrite core and (ii) periphery of the secondary dendrite lobes (as indicated in Fig. 2) to determine the as-cast micro-segregation. In the case of (i), freezing of the primary dendrite core marks the start of solidification $\left(f_{S}=0\right)$; likewise in the case of (ii) the freezing of the periphery of the secondary dendrite lobe that distinguishes the dendrite morphology from the inter-dendritic constituent occurs at a given temperature. This is because the freezing of the inter-dendritic constituent commences only when the liquid composition reaches a critical value, as schematically illustrated for a binary system in Fig. 3. We further emphasize that in both cases the extent of scatter should be minimum, since all locations (i) have solidified at similar temperatures and likewise for all locations (ii). Specifically at the lobe periphery, we have offset the measurement points from the inter-dendritic region, so that we remain within the dendrite. The proximity of the points from the lobe perimeter $(d)$ was $5 \mu \mathrm{m}$; this is much smaller than one-half the primary dendrite spacing $\left(1 / 2 \lambda_{P} \sim 150 \mu \mathrm{m}\right)$. As an estimate, the series of measurement points can also be construed as belonging to a contour of nearconstant $f_{S}$, since this offset can be seen to have a minimal effect on fraction solid, $f_{S}$, i.e. $\Delta f_{S} \approx 5 / 150=0.03$. Therefore, the measurement points on the secondary lobe periphery would have solidified at a similar temperature.

Accordingly, the measured compositions at the dendrite cores and at the lobe periphery for the principal (refractory) solute species are as follows:

$$
\begin{gathered}
\mathrm{C}_{\mathrm{Ta}}=[6.59 \pm 0.07] \text { wt. } \%, \mathrm{C}_{\mathrm{W}}=[7.35 \pm 0.07] \text { wt. \% } \\
\mathrm{C}_{\mathrm{Re}}=[9.58 \pm 0.28] \text { wt. } \% \\
\text { - Measured at dendrite Cores } \\
\mathrm{C}_{\mathrm{Ta}}=[8.50 \pm 0.24] \text { wt. } \%, \mathrm{C}_{\mathrm{W}}=[4.79 \pm 0.23] \text { wt. \% } \\
\mathrm{C}_{\mathrm{Re}}=[5.26 \pm 0.44] \text { wt. } \%
\end{gathered}
$$

- Measured at dendrite lobe peripheries

We note that the measured scatter in measurements is comparable with the uncertainty of measurement, $\delta$, which is an instrumental parameter. Specifically:

$$
\delta_{\mathrm{Ta}}=0.10 \text { wt. } \%, \delta_{\mathrm{W}}=0.13 \text { wt. } \%, \delta_{\mathrm{Re}}=0.22 \text { wt. } \%
$$

Clearly, Ta partitions to the liquid, while both $\mathrm{W}$ and Re partition to the solid and are consistent with other studies. The extent of back-diffusion in the solid during solidification is dependent on the local solidification time. Therefore the measured composition at the dendrite core in the solid-state is expected to have a greater contribution from back-diffusion, than the measured composition at the lobe periphery. This is equivalent to stating that the measured dendrite core composition does not correspond to a point on the solidus with the consequence that it is erroneous to deduce partition coefficients at the dendrite tips from as-cast microstructures However, the lobe periphery corresponds to the latter stages in solidification and back-diffusion now is only limited from this temperature to the solidus, since diffusion in the fully solid-state, i.e. when solidification is complete is minimal. Therefore, the measured composition at the lobe periphery lies closer to the actual solidus curve. The important aspect that follows directly from this section is the extent of coring in the ascast microstructure, which consequently determines the amount of the non-equilibrium inter-dendritic constituent that directly has a bearing on the heat treatment process mentioned in the introduction.

Therefore, from the onset of solidification to the end of primary solidification the extent of compositional heterogeneity across the dendrite cross-section is as follows:

$$
\Delta \mathrm{Ta}=1.9 \text { wt. } \%, \Delta \mathrm{W}=2.6 \text { wt. } \%, \Delta \operatorname{Re}=4.3 \text { wt. } \%
$$

We note that other authors have also reported the variation of solute composition with fraction solid. The method adopted there involved an in-depth analysis of the measured solute composition on as-cast microstructures using the commonly used point-count method. Subsequent sorting of the measured composition using different algorithms yields the evolution of solute composition with fraction solid $[2,13]$. However, the primary limitation in all those measurements is the appreciable bias that arises from the volume averaging effect, which we have discussed. This has been suppressed in our measurements thus giving a more reliable estimate of solute segregation.

\section{Identification of Solidification Sequence}

In section 3.1, we have presented features of interest in the enthalpy and effective heat capacity curves. The extent of microssegregation reported above has a direct bearing on the resultant solidification sequence, which will be discussed here in relation to the DSC data.

The primary solidification path corresponds to freezing of the $\gamma$ phase, which constitutes the dendrites. This is also predicted by the $E Q L B$ model in $\mathrm{JMatPro}^{\mathrm{TM}}$ and can be represented as:

$$
\mathrm{L}_{1} \rightarrow \gamma \quad 1365^{\circ} \mathrm{C}<\mathrm{T} \leq 1406^{\circ} \mathrm{C}
$$

As solidification proceeds, $\gamma$ phase grows into dendritic morphology. $\mathrm{W}$ and Re segregate to the $\gamma$ dendrites, while Ta, Ti and $\mathrm{Al}$ are enriched in the remaining liquid. However, $\mathrm{T}=1365^{\circ} \mathrm{C}$ corresponds to the end of primary solidification.

A high magnification secondary electron image (SEI) that focuses on the inter-dendritic constituent enclosed by the secondary dendrite arms is shown in Fig. 4a. Here the $\gamma^{\prime}$ has been etched and therefore the resultant contrast is one of topography. More highresolution images using the InLens detector (section 2.2) that show the microstructures with enhanced clarity and detail are presented in Figs. 4b, c respectively. 

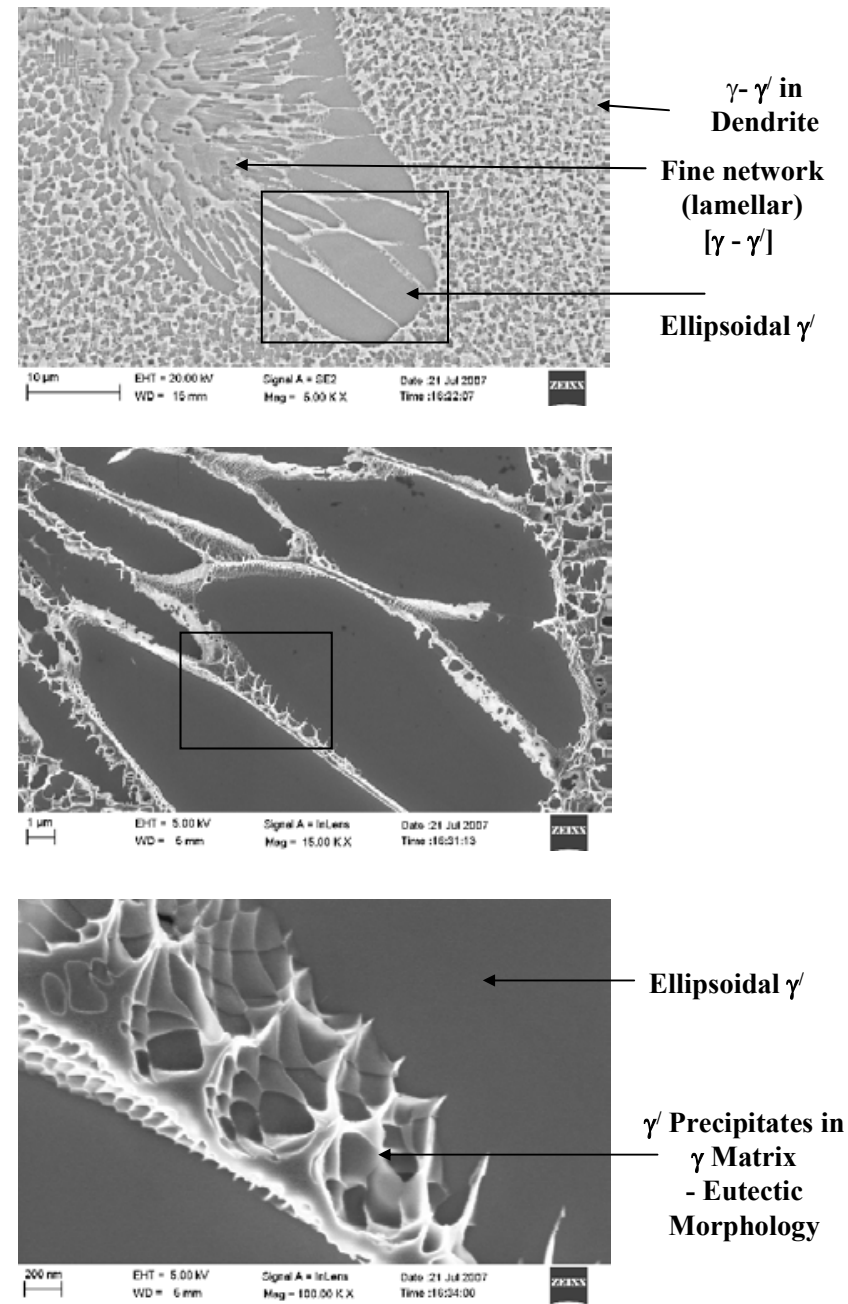

Fig. 4 Secondary electron images (SEI) focusing on the periphery of the secondary dendrite lobes and inter-dendritic region: (a) low magnification, (b) and (c) high magnification images using InLens detector showing details of the region between impinging ellipsoidal $\gamma^{\prime}$ precipitates in the inter-dendritic region.

Three distinct morphologies are observed:

i. $\quad$ Regular $\gamma-\gamma^{\prime}$ microstructure within the dendrite $\left(\gamma^{\prime}\right.$ phase precipitates coherently from the $\gamma$ dendrites when the sample was further cooled below the $\gamma^{\prime}$-solvus, i.e. in the solid-state).

ii. Coarse $\gamma$ particles having ellipsoidal morphology that are separated by a channel when they impinge.

iii. Fine network type $\left[\gamma-\gamma^{\prime}\right]$ morphology that appears to emanate from the coarse $\gamma$.

The inter-dendritic constituent is comprised of morphologies (ii) and (iii). There is an important aspect here, which we explain below:

Size Consideration - This is clearly seen in the micrographs in Figs. $4 \mathrm{~b}$, c. The deep-etched microstructure in Fig. $4 \mathrm{~b}$ shows that the channel mentioned in (ii) above, which is where the ellipsoidal $\gamma /$ precipitates impinge, actually comprises of a discrete $\gamma / \gamma^{\prime}$ distribution. In fact the high magnification image in Fig. 4c shows this layer more clearly, where the $\gamma^{\prime}$ precipitates within this layer are clearly seen. With this observation in perspective and with reference to Fig. 4a, we therefore propose that the fine network lamellar $\left[\gamma-\gamma^{\prime}\right]$ morphology, referred to as (iii) actually corresponds to the sandwiched section enclosed between coarse $\gamma$ particles; however here the $\gamma^{\prime}$ particles are spatially located above and below the observation plane. This is unlike in case (ii), where we are observing an oblique section through the $\left[\gamma-\gamma^{\prime}\right]$ network, with the result that the lamellar network now appears as a channel with the impinging $\gamma /$ particles on either side.

We now come to the second aspect, which is as follows:

Thermodynamic Consideration - Clearly from the morphology therefore it follows that this sandwiched layer comprises of the eutectic product that occurs at the terminal stages of solidification. Now if the coarse (ellipsoidal) $\gamma /$ particles are also the product of a eutectic reaction $\left(\mathrm{L} \rightarrow \gamma+\gamma^{\prime}\right.$ ), then subsequent solidification cannot be followed by another two-phase eutectic reaction $(\mathrm{L} \rightarrow \gamma$ $\left.+\gamma^{\prime}\right)$ to form the fine $\left[\gamma-\gamma^{\prime}\right]$ morphology.

Although in most studies morphologies (ii) and (iii) are lumped together and characterized as eutectic, the evolution of these microstructures follows a definite sequence and needs careful addressing. We propose therefore that the coarse (ellipsoidal) $\gamma^{\prime}$ particles are the product of a peritectic reaction instead and preceding the eutectic reaction. In addition to the micrographs in Figs. $4 \mathrm{a}-\mathrm{c}$ and the thermodynamic argument presented above, another evidence for this reaction is given:

Morphological Consideration - A convex shape (view from $\gamma$ dendrite) of the interface between $\gamma^{\prime}$ and $\gamma$ dendrite is observed in Fig. 4b, which shows the interface between the dendrite periphery and the ellipsoidal $\gamma^{\prime}$ particles in more detail. This clearly reveals that $\gamma^{\prime}$ phase grows into the primary $\gamma$ dendrites; this can occur only if the ellipsoidal $\gamma^{\prime}$ particles are the product of a peritectic reaction.

We therefore propose that the onset of freezing of the interdendritic region constitutes the following reaction:

$$
\mathbf{L}_{2}+\gamma \rightarrow \gamma^{\prime} \quad \mathbf{T}^{\prime}<\mathbf{T} \leq \mathbf{1 3 6 5}^{\circ} \mathrm{C}
$$

This sequence is also borne by thermodynamic calculations performed by Warnken et al [14] for the alloy CMSX4. However, it is interesting to note that thermodynamic calculations using the SCHEIL solidification model in JMatPro ${ }^{\mathrm{TM}}$ only predicts the eutectic transformation and therefore cannot account for the morphology in Figs. $4 \mathrm{a}-\mathrm{c}$. Solidification terminates with the eutectic reaction:

$$
\mathbf{L}_{3} \rightarrow \gamma+\gamma^{\prime} \quad \mathbf{T}^{\prime} \leq \mathbf{T} \leq \mathbf{1 3 5 8}^{\circ} \mathbf{C}
$$

\section{Representation of Solidification Sequence}

Having proposed the solidification sequence above, we now explain this sequence using a space model so that the various transformations can be visualized. For this purpose we take recourse to a $3 \mathrm{D}$ space model. Owing to the inadequacy of the thermodynamic database, we represent multi-component CMSX10K as a pseudo-ternary system. Such an approach has 

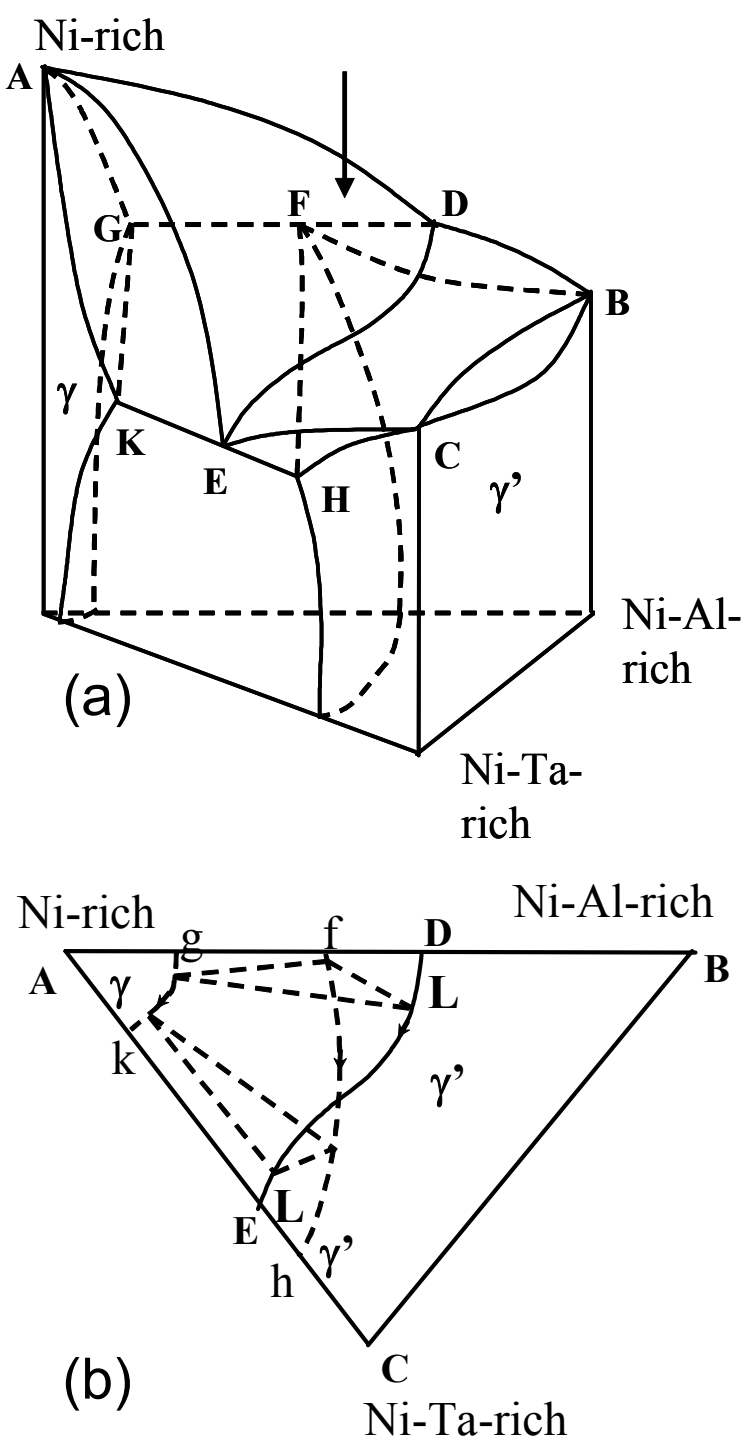

Fig. 5 Schematic representation of solidification sequence using a pseudo-ternary phase diagram: (a) Three-dimensional space model, (b) Horizontal section at a given temperature showing tie triangles and projection of liquidus and solidus curves.

been successively adopted by other authors in the analysis of superalloys $[15,16]$. The only phases that we consider are $\mathrm{L}, \gamma$ and $\gamma^{\prime}$. In Fig. 5a the required space model is shown [17]. The three binaries shown comprise of a peritectic reaction, a eutectic reaction and in the third case there exists complete solubility. In Ni-base superalloys, which are multi-component, it would be appropriate to represent one corner, i.e. A as Ni-rich (solvent) and this is indicated in Fig. 5a and would represent the $\gamma$-phase field. Therefore, the $\gamma^{\prime}$ phase should encompass the solute-rich corners of the phase diagram, i.e. B and C. The Ni-Al binary system shows a peritectic reaction and a eutectic reaction; the former occurring at a higher temperature $\left(\approx 1.5^{\circ} \mathrm{C}\right)$ and at a lower fraction Al [15]. Consequently we label corner $\mathrm{B}$ as Ni-(Al-rich). Therefore, the third corner C, should also be a $\gamma^{\prime}$-stabilizer. Either $\mathrm{Ti}$ or $\mathrm{Ta}$ are alternatives. We choose $\mathrm{Ni}-\mathrm{Ta}$ (rich), since
CMSX10K is a high Ta containing alloy. Further we note that the Ni-Ta binary system shows a eutectic reaction [18] We wish to emphasize here that we have made no attempt to indicate compositions; all that we state is that the edges $\mathrm{AB}, \mathrm{BC}$ and $\mathrm{CA}$ do not correspond to simple binaries as one would expect in a ternary phase diagram, rather we have a pseudo-ternary system. It follows therefore, that corners A, B and C do not represent $100 \%$ A, $100 \%$ B and $100 \%$ C respectively. Such a space model will facilitate understanding the solidification sequence that we have proposed on thermodynamic considerations and that is supported by the as-cast microstructure.

From Fig. 5a, we identify the following:

- $\quad$ Liquidus surfaces - AED and BCED

- $\gamma$-solidus surface - AGK; $\gamma^{\prime}$-solidus surface - BCHF

Since under equilibrium conditions only primary solidification occurs $(\mathrm{L} \rightarrow \gamma)$, the nominal composition, $\mathrm{C}_{0}$ lies within $\mathrm{AGK}$, as shown in Fig. $5 \mathrm{~b}$, which shows the projections of the various curves in Fig. 5a on a representative tie-triangle However, owing to restricted back-diffusion in the solid, the $\gamma$-solidus is depressed, as seen schematically in Fig. 3 for a binary system, because of which solidification does not terminate at the solidus; rather the liquid composition, $\mathrm{C}_{\mathrm{L}}$ reaches the eutectic point. A similar situation is encountered here, where because the solidus surface is depressed; $\mathrm{C}_{0}$ now lies outside the region AGK, i.e. not within the primary phase field.

Keeping in mind the solidification sequence proposed in the preceding section, the following features are pertinent:

\section{Primary Solidification:}

The liquid composition moves along AED, while the corresponding composition of the $\gamma$ phase traverses the surface AGK. The exact positions of the extremities of the tie-lines, i.e. L and $\gamma$ compositions would have to be determined experimentally using quenched experiments.

\section{Freezing of Inter-Dendritic Constituent:}

The onset of freezing of the inter-dendritic region commences with the peritectic reaction.

(a) Peritectic: At this point the liquid composition reaches curve DE. Simultaneously, $\gamma$ and $\gamma^{\prime}$ compositions reach curves GK and HF respectively thus defining the tie-triangle such as in Fig. $5 \mathrm{~b}$, but at the peritectic temperature. As the three-phase peritectic reaction proceeds, the respective phase compositions traverse along the curves as marked with arrowheads in Fig. 5b.

(b) Eutectic: However, a cross-over of the liquidus curve DE occurs with respect to the $\gamma^{\prime}$-solidus curve HF and this marks the start of the eutectic reaction. The positions of the compositions on the new tie-triangle are marked and solidification terminates when the nominal composition, $\mathrm{C}_{0}$ lies on the $\gamma-\gamma^{\prime}$ side of the tie-triangle.

This solidification sequence of the inter-dendritic constituent differs slightly from that proposed earlier [19]. In that study it was suggested that following the peritectic reaction but preceding the eutectic reaction, growth of $\gamma^{\prime}$ occurs in the liquid, as indicated by: 
$\mathrm{L} \rightarrow \gamma^{\prime}$. Such a scheme cannot be expressed in a ternary system, as seen from Figs. 5a, b, albeit it can occur in a binary system.

In a normal ternary system, from knowledge of the tie-triangle compositions and the nominal composition, $\mathrm{C}_{0}$, it is straightforward to calculate the extent of occurrence of the various phases from a simple centre-of-gravity method. However, since we were only considering pseudo-ternary approximations to graphically visualize the phase transformations, an alternate method must be used to determine the amount of inter-dendritic (non-equilibrium) constituent in the as-cast microstructure. This will be calculated using the calorimetry approach as outlined below.

\section{Evolution of Fraction Solid}

The measured enthalpy in Fig. 1a will be used to calculate the fraction solid. Fig. 6 shows a schematic representation of the enthalpy $(H)$ versus temperature $(T)$. If we assume $C_{P, \mathrm{~S}}=C_{P, L}=$ $C_{P}$, (solid and liquid phases have equal heat capacities) then by extrapolating the pure liquid and solid enthalpies as in Fig. 6, we obtain the following expressions:

For the pure solid:

$$
H_{S}=\left[H_{\text {Solidus }}+C_{P, S}\left(T-T_{\text {Solidus }}\right)\right]
$$

For the pure liquid:

$$
H_{L}=\left[H_{\text {Liquidus }}+C_{P, L}\left(T-T_{\text {Liquidus }}\right)\right]
$$

The enthalpies $H_{S}$ and $H_{L}$ refer to the enthalpy per unit mass for the compositions that exist in the two phases and not the initial composition. For the mixture of solid and liquid:

$$
H=f_{S} H_{S}+\left(1-f_{S}\right) H_{L}
$$

The weight fraction solid $\left(f_{S}\right)$ can then be calculated as:

$$
f_{S}=\left(H_{L}-H\right) /\left(H_{L}-H_{S}\right)=\left(H_{L}-H\right) / L
$$

where $L$ is the latent heat and equals $\left[H_{L}-H_{S}\right]$ and is constant (Fig. 6). Therefore, using equation 2 the fraction solid $\left(f_{S}\right)$ can be determined. However, as solidification progresses there are changes in the composition of the solid and liquid and hence enthalpy is a function of composition as well as temperature. It has been shown in a previous study that in Ni-base superalloys the compositional contribution to the latent heat is small and can be neglected [20]. Therefore, equation (2) is a valid approximation.

The evolution of fraction liquid, $f_{L}\left(1-f_{S}\right)$ is presented in Fig. 7a. The recalescence in the enthalpy curve is reproduced well in this calculation. Both at the start of solidification as well as at the onset of the peritectic transformation indicating the commencement of freezing of the inter-dendritic constituent the nucleation undercooling is manifested. Corresponding to Fig. 1b and section 3.1, the onset of freezing of the inter-dendritic region corresponds to $T=1365^{\circ} \mathrm{C}$. From Fig. 7a, the corresponding fraction liquid is $f_{L}=0.9$ and this corresponds to the mass fraction of the inter-dendritic region.
It is a common practice to approximate the solidification kinetics with the Scheil model. Using the latter approach we have obtained the fraction liquid using JMatPro ${ }^{\mathrm{TM}}$. This is superimposed on the calculations using the DSC method in Fig. 7b where we have focused on the latter stages of solidification in the vicinity of the freezing of the inter-dendritic constituent. It is clearly seen in Fig. 7b that $f_{L}$ is significantly over-estimated and therefore the Scheil approach is not suitable in such calculations. A similar calculation in an earlier generation Ni-base superalloy, IN713LC shows a similar result [20].

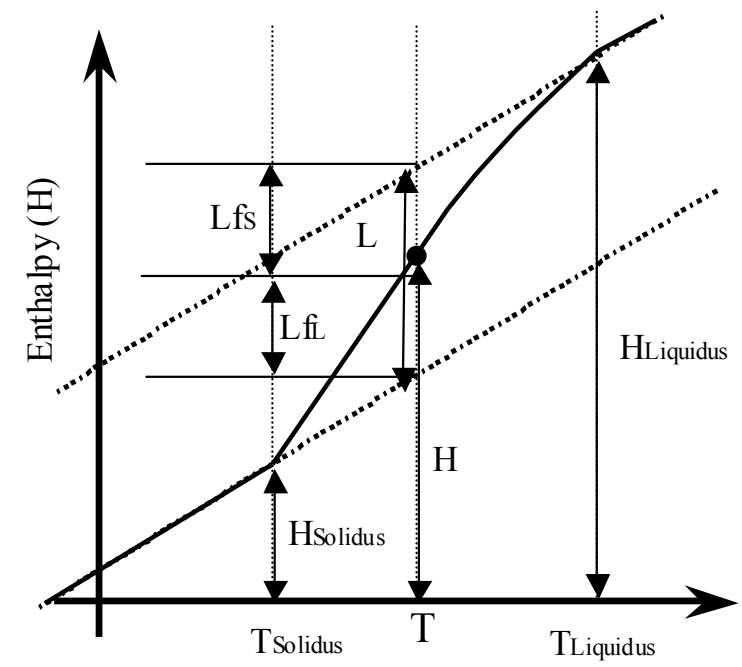

Temperature $(\mathrm{T})$

Fig. 6 Schematic diagram indicating the principles in the calculation of evolution of fraction solid from measured enthalpy.
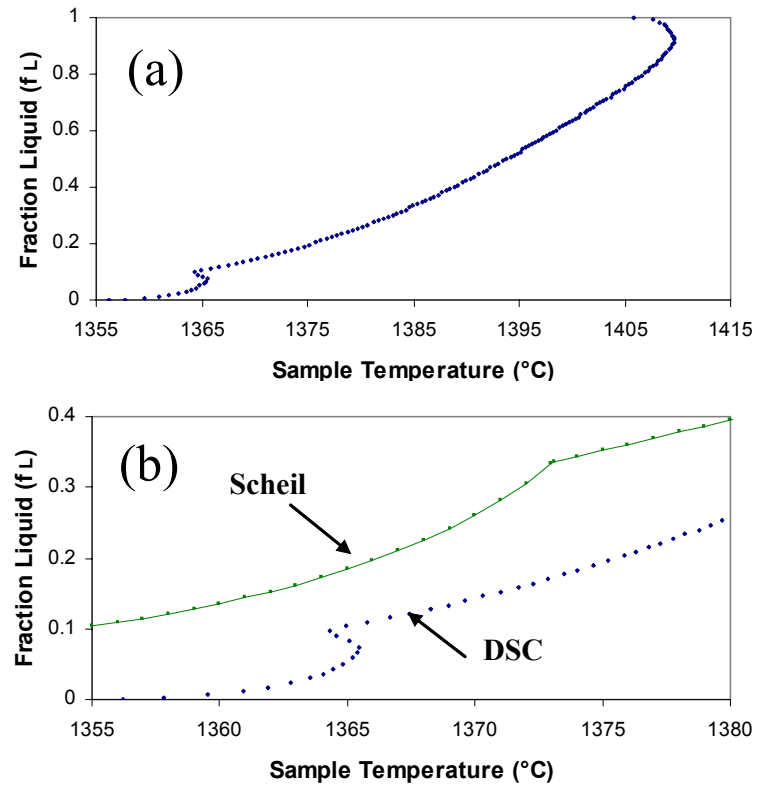

Fig. 7 Evolution of fraction liquid versus temperature: (a) Calculated from measured enthalpy using DSC, (b) Comparison with Scheil calculations and focusing on latter stages of solidification. 
Applications of this Study

Keeping in mind that the motivation of this study was to provide relevant parameters in the design of an optimum heat treatment cycle as outlined in the introduction, we summarize below the key results and highlight their application.

\section{Identification of Solidification Sequence}

We have clearly demonstrated the solidification sequence accompanying the freezing of the inter-dendritic region, which characterizes the non-equilibrium phases that form. Additionally, we have obtained the temperature at which the onset of freezing of this region occurs as well as the temperature at which solidification terminates; the latter correlating well with the incipient melting temperature. Therefore, based on this study we recommend that to aid the dissolution of the non-equilibrium inter-dendritic constituent during heat treatment by facilitating liquid state diffusion, an upper bound for the isothermal hold temperature during the solutioning stage should be: $T=1365^{\circ} \mathrm{C}$. At this temperature the non-equilibrium phases will melt, if they exist within the microstructure, but during the isothermal hold, diffusion occurs and the primary $\gamma$-solid (dendrites) grows into the liquid. Nevertheless, a temperature nearer the upper bound rather than the incipient melting point is optimum given the arguments above, which will result in the growth of the primary solid when the melted liquid disappears during a prolonged isothermal hold.

\section{Extent of Micro-segregation}

Prior to ageing, dissolution of the non-equilibrium phases as well as compositional homogeneity across the dendrite cross-section is mandatory. Methods commonly used involving X-ray spectroscopy on as-cast microstructures in Ni-based superalloys have an associated error that arises from volume averaging, since there is an appreciable variation in the $\gamma /$ precipitate size across the dendrite cross-section arising from micro-segregation of $\mathrm{Ta}, \mathrm{W}$ and Re. This artefact was overcome using the method adopted in this study by heating to above the $\gamma^{\prime}$-solvus temperature in the solid-state followed by a rapid quench. An increase in Ta (1.9 wt. \%), while a decrease in W (2.6 wt. \%) and Re (4.3 wt. \%) concentrations from the dendrite core to the dendrite lobes was measured.

\section{Amount of Non-Equilibrium Phases (Inter-Dendritic)}

While (I) indicates an upper bound of temperature and (II) indicates the extent of compositional heterogeneity, the duration of the isothermal hold depends on the amount of the interdendritic constituent. Using the enthalpy-based method the corresponding mass fraction was: $f_{L}=0.9$.

\section{Concluding Remarks}

A method has been presented to evaluate the solidification characteristics of an alloy. From knowledge of the quantities measured and calculated above, computational models such as DICTRA or ThermoCALC can then be used to derive an optimum isothermal hold at a given temperature during heat treatment to produce a homogeneous microstructure. The method is simple and general in its validity and is a potential tool in assessing castability and heat treatment in exploratory alloy design.

\section{Acknowledgements}

The authors express gratitude to their colleagues who have contributed to these studies. These include Dr. H. Stone and Dr. H. T. Pang, Department of Materials, University of Cambridge, UK, Dr. C. L. Hayward, School of GeoSciences, University of Edinburgh, UK, Dr. B. A. Shollock, Mr. M. Lekstrom, Department of Materials, Imperial College, London, UK, Mr. B. Kantor, Precision Foundry (Bristol), Rolls-Royce plc, UK, Mr. Steve Wardle, Bodycote Ltd., (Derby), UK. One of the authors, H. B. Dong acknowledges the Royal Society and EPSRC for offering him a Royal Society Industry Fellowship and Rolls-Royce ple for hosting him.

\section{References}

1. R. C. Reed, The Superalloys Fundamental and Applications (Cambridge University Press, 2006), 2.

2. R. A. Hobbs, S. Tin and C. M. F. Rae, "A Castability Model on Elemental Solid-Liquid Partitioning in Advanced Ni-Base Single Crystal Superalloys," Metallurgical and Materials Transactions, 36A (2005), 2761 - 2773.

3. T. T. Field et al., Heat Treatment of Single Crystals (United States Patent, Patent Number 4,583,608, April 22, 1986).

4. S. R. Hegde, R. M. Kearsey and J. C. Beddoes, "Resolidification Behaviour of Single-Crystal Superalloys," Scripta Materialia, 57 (2007), 837 - 840.

5. F. Tancret, "Thermo-Calc and DICTRA Simulation of Constitutional Liquation of Gamma Prime during Welding of Ni-Base Superalloys," Computational Materials Science, 41 (2007), 13 - 19.

6. H. B. Dong and J. D. Hunt, "A Numerical Model for a Heat-Flux DSC: Determining Heat Transfer Coefficients within a DSC," Materials Science and Engineering, A 413414 (2005), $470-473$.

7. M.J. Goulette, P.D. Spilling and R.P. Arthey, "Cost Effectrive Single Crystals," Superalloys 1984, ed. M. Gell et al. (Warrendale, PA: The Metallurgical Society of AIME, 1984), 167 - 176.

8. H. B. Dong and R. Brooks, "Determination of Liquidus Temperature in Al-Si and Al-Si-Mg alloys using a SinglePan Scanning Calorimeter," Materials Science and Engineering, A 413-414 (2005), 480 - 484.

9. R. I. Wu and J. H. Perepezko, "Liquidus Temperature Determination in Multicomponent Alloys by Thermal Analysis," Metallurgical and Materials Transactions, 31A (2000), $497-501$.

10. W. J. Boettinger and U. R. Kattner, "On Differential Thermal Analyzer Curves for the Melting and Freezing of Alloys," Metallurgical and Materials Transactions, 33A (2002), $1779-1794$. 
11. Z. Guo, N. Saunders, A. P. Miodownik and J-Ph Schille, "Modelling of Materials Properties and Behavior Critical to Casting Simulations, Materials Science and Engineering, A 413-414 (2005), 465 - 469.

12. N. D'Souza, M. Lekstrom and H. B. Dong, “An Analysis of Measurement of Solute Segregation in Ni-Base Superalloys using X-Ray Spectroscopy", Materials Science and Engineering $A$ (in press - DOI information: 10.1016/j.msea.2008.01.025).

13. M. Ganesan, D. Dye and P. D. Lee, "A Technique for Characterizing Microsegregation in Multicomponent Alloys and its Applications to Single-Crystal Superalloy castings", Metallurgical and Materials Transactions, 36A (2005), $2191-2204$.

14. N. Warnken et al., "Investigation of Eutectic Island Formation in SX Superalloys", Materials Science and Engineering, A 413-414 (2005), 267 - 271.

15. M. Durrand-Charre, The Microstructure of Superalloys (Gordon and Breach Science Publishers, Amsterdam, The Netherlands, 1997), 15 - 33.

16. J. N. Dupont, C. V. Robino and A. R. Marder, "Modelling Solute Redistribution and Microstructural Development in Fusion Welds of Nb-Bearing Superalloys", Acta Materialia, 46 (1998), 4781 - 4790.

17. D. R. F. West and N. Saunders, Ternary Phase Diagrams in Materials Science (Maney Publishing, Institute of Materials, UK, $3^{\text {rd }}$ Edition, 2002), 51.

18. Metals Handbook, Metallography, Structures and Phase Diagrams (Vol. 8, $8^{\text {th }}$ Edition, ASM, Metals Park, Ohio, 1973).

19. N. D'Souza and H. B. Dong, "Solidification Path in High Refractory Containing Third Generation Ni-Base Superalloys, with an emphasis to Last Stage Solidification," Scripta Materialia, 56, (2007), 41 - 44.

20. N. D'Souza et al., "Quantitative Characterisation of Last Stage Solidification in a Ni-base Superalloy using an Enthalpy-Based Method," Materials Science and Technology, 23 (2007), 1085 - 1092. 